\title{
2
}

\section{Integrated Customer-Focused Network Management: Architectural Perspectives}

\author{
L. Feldkhun, M. Marini, S. Borioni \\ Sodalia SpA \\ Via V. Zambra 1, 38100, Trento, Italy, Fax +39461316401
}

\begin{abstract}
The industry-wide trend to decouple telecommunications services from the underlying network technology, together with increasing complexity of high-speed hybrid customer networks, has created the need for a new generation of integrated network management systems, dedicated to managing advanced heterogeneous customer networks as a service.

This paper presents business drivers for Integrated Customer-Focused Network Management (ICFNM) systems and introduces a number of key architectural perspectives for ICFNM implementation including: i) the position of ICFNM systems in the overall network management context, ii) the functional scope, and iii) the practical experience gained in designing and developing ICFNM using Object Oriented methodology and advanced commercial technologies (i.e., CORBA).
\end{abstract}

\section{Keywords}

Integrated Network Management, CNM, Architecture, CORBA

\section{INTRODUCTION: ROLE AND POSITION OF ICFNM}

The ongoing rapid technological innovation and deregulation in the telecommunications industry creates business opportunities for telecommunications network and service providers. One area where these opportunities are greatest is in offering business customers an increasingly high level of control of network resources and services at decreasing costs.

This can be achieved by Virtual Private Networks (VPN): a service offering which allows business clients of Public Telecommunications Operators (PTO) to create corporate networks and define telecommunications services based on public network resources. 


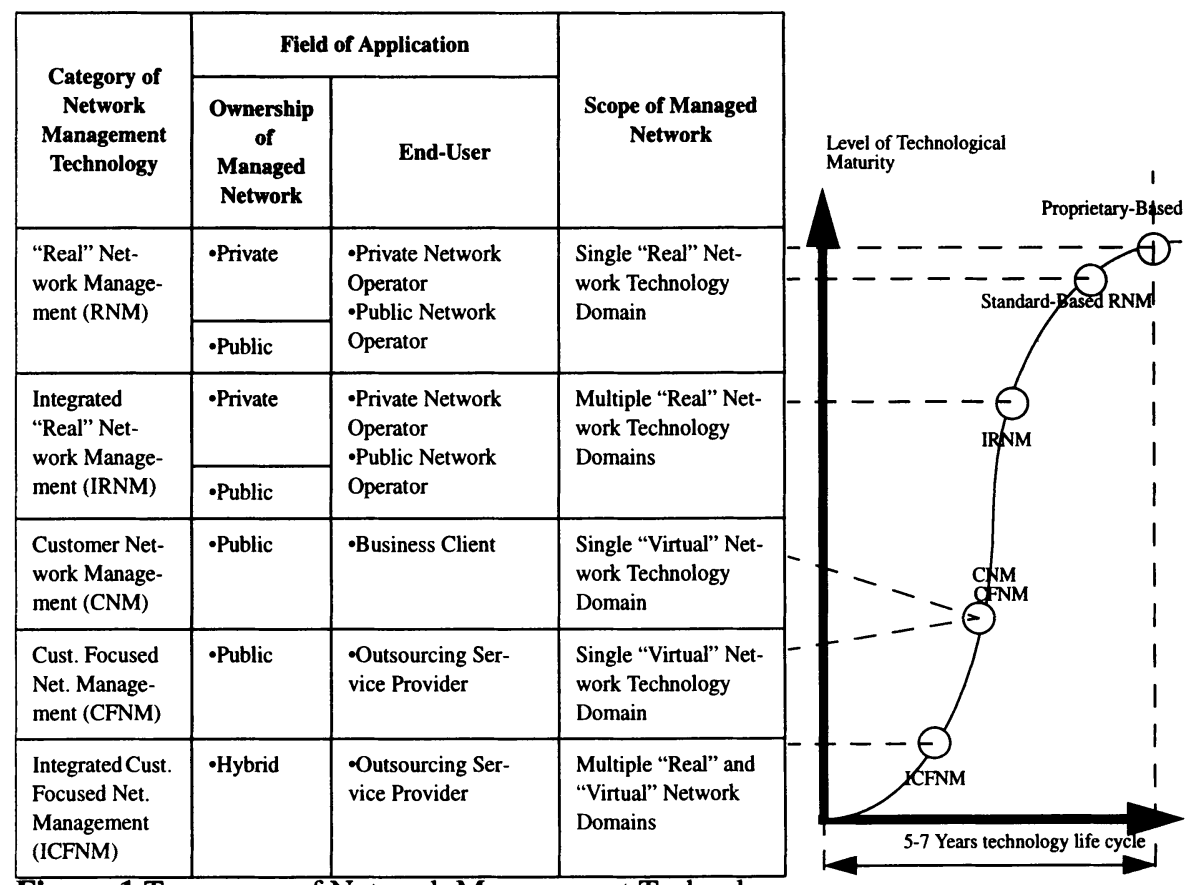

Figure 1 Taxonomy of Network Management Technology.

Additionally, control can be offered through Outsourcing Services, which include a wide range of contractually defined services covering Operations, Administration, Maintenance and Provisioning of hybrid (in terms of ownership) corporate networks according to defined Service Level Agreements.

The growth of VPNs and Outsourcing business is strongly increasing because of:

- increasing role of telecommunication technology in business customers' competitive position

- rapid pace of technological innovation in telecommunications

- increasing technological sophistication and required skill level for telecommunications management

- increasing risk of technological obsolescence of privately owned telecommunications assets

- increasing level of customer focused control of publicly offered telecommunications resources and services (Bigaroni, 1992)

- decreasing costs of alternatives to private networks.

Provisioning for sophisticated services require network operators to introduce new generations of management systems and applications (Figure 1).

In this context we introduce the notion of an Integrated Customer Focused Network Management (ICFNM) system (Feldkhun, 1996). This system provides a particular view of network/service management, reflecting the perspective of a telecommunications services outsourcing contract established between a business client and an outsourcing service provider. The network and service management related information 
and controls, available to the end-user of ICFNM, are limited to the public network resources and services, which are contractually allocated to a particular customer of a PTO provider. This is in contrast to the amount of management information and the level of control available to the end-user of the "Real" Network Management (RNM) system of the public network. An ICFNM system generates and maintains a customer focused view of the status of resources/services in a multidomain (in terms of technology and ownership) network.

\section{CHALLENGES FOR AN ICFNM SYSTEM}

The objectives of ICFNM can be better understood by examining the context in which the system is positioned. Context analysis contributes to identify challenges for ICFNM and key architectural requirements for an effective system design.

\subsection{Managed Networks}

The need to deploy highly sophisticated communication services is reflected in the complexity of communication networks which are built on a number of interconnected transmission (e.g. PDH, SDH) and switching (e.g., X.25, Frame Relay, ATM) technologies. Each network technology is potentially offered by multiple suppliers.

Network and service operators are required to implement effective management to control and monitor network systems from a customer point of view in order to meet customers expectations and changing needs.

The main challenge is to provide operators with a complete and integrated view of the complex managed network environment. As an example of the network complexity, let's think of the number of equipment and network technologies which are crossed by an Internet Protocol (IP) packet generated at a desktop workstation. It crosses customer routers, it is encapsulated, for instance, into a Switched Multimegabit Data Service (SMDS) Protocol Data Unit, segmented into ATM cells, and it is then transmitted via a permanent Virtual Path Connection (VPC). The VPC may be physically laying on top of a PDH circuit multiplexed by access multiplexers, "SDH crossconnected" to finally reach a SMDS switch, which will terminate the ATM VPC and route the packet and so forth until the packet destination is reached.

From this complex and heterogeneous network stems the requirement for ICFNM to be capable of fulfilling operator needs for a simple-to-use tool; the tool allows for an integrated Operation Administration and Management (OA\&M) of the end-to-end connectivity across multiple network technologies.

\subsection{Management domains}

Definitions of management domains have been extensively explored in the industry (Sloman, 1994).

The need to address multiple management domains by ICFNM is meant in terms of network ownership. Virtual Private Networks span customer premises and wide area 
networks (Figure 2), where customer premises equipment may be owned and managed by the customer or, alternatively, outsourced to PTO or other suppliers of outsourcing services.

Wide area networks are sectioned in different network domains under the control of different, sometime competing, network operators. Within one single PTO, different organizations may control different parts of the whole PTO network.

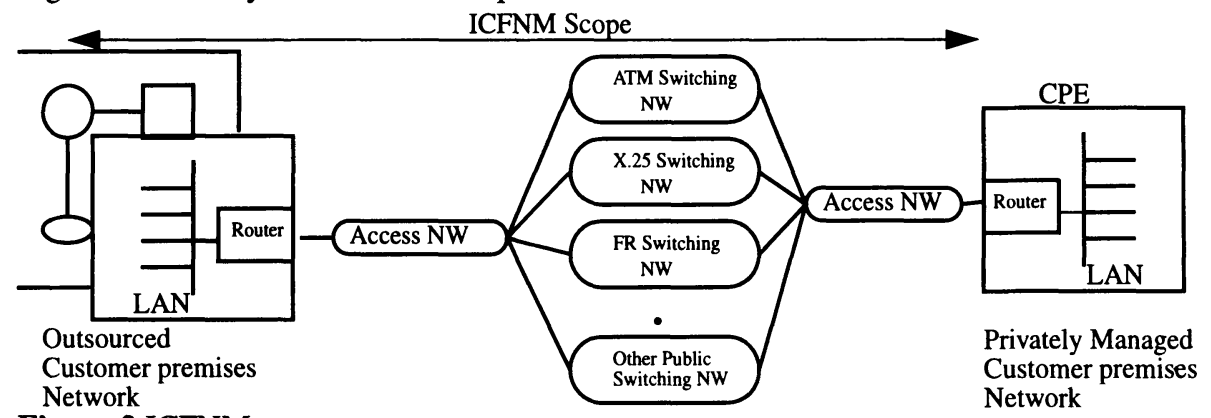

Figure 2 ICFNM management scope.

While customer premises equipment and networks are fully dedicated to a given customer, network resources in the wide area network domain (e.g. access networks, multiplexers, switches, trunks, ATM Virtual Paths, SDH Virtual Containers) are generally shared among many of them. In the context of ICFNM, network resources are filtered and processed in order to find out those which are relevant to the customer being managed. From this perspective, for instance, a single ATM VP may belong to multiple virtual networks and therefore be monitored as a network resource virtually owned by multiple customers.

From these considerations originates the requirement for ICFNM to virtualize and integrate management domains consisting of resources under different ownership. The scope of ICFNM applications and the types and characteristics of available management operations are discussed in section 3.

\subsection{Network Element and Network Management systems}

Achieving an integrated view of the overall end-to-end network (from a customer perspective) requires ICFNM to interoperate with a set of Network Elements (NE) and Network Management Systems (i.e. "Real" Network Management System), which are designed and developed according to various criteria. This happens not only in case of different managed technologies, but also for managing equipment of the same technology but provided by different vendors.

Lack of standards for network management information models has caused a proliferation of proprietary solutions for the systems deployed in the market. Information models defined by ITU-T are available for a set of technologies, aimed at the public network side - a most prominent example is represented by SDH. Nevertheless, the late availability of the standards coupled with the time-to-market pressure has caused manufacturers to take proprietary approaches (e.g. adopting early drafts of the standards).

On the other side, Internet has defined, early on, a number of management "informa- 
tion models" (e.g. MIB 2 and extensions) complementary to the ITU-T ones, which have been successfully deployed in the field, but have limitations in covered network technologies and services.

As a result, a significant number of the network elements deployed in the field currently support proprietary management interfaces, and protocols. The need to integrate these networks has led, in some cases, to the development of SNMP and CMIP proxies providing views at either Network or NE level.

Peculiarities of management systems are not restricted to the aforementioned elements. Often NE and Network Management systems come with built-in limitations, mainly due to the fact that such systems have not been designed for further integration with other systems and/or applications.

Therefore almost every management system is restricted in some capabilities relevant to ICFNM, ranging from performance (e.g. response time to an external request), scalability (e.g. possibility to scale in order to serve a large number of application requests) and configuration possibilities (e.g. number of destination addresses for forwarding an asynchronous notification). For this reason the ICFNM architecture needs to address the differences in data models, protocols and functional capabilities offered by the interfaced managements systems.

\subsection{Operation Support Systems}

ICFNM capability to interoperate with a set of NE and Network Management systems is necessary, but not sufficient to achieve Integrated Customer Focused Network Management. Additional data, not available at the Real Network Management (RNM) level, is needed in order to model inter-network and service configurations.

From an architectural point of view ICFNM obtains additional information, complementing what is available at a network and network management level, from a set of Operation Support Systems (OSSs). Examples of OSSs relevant to the ICFNM application context are:

- Customer Management OSS (Contract OSS)

- Billing OSS

- Service Provisioning OSS

- Trouble Ticketing OSS (TT OSS).

For example, for an Outsourcing Business a Customer Management OSS will supply ICFNM with customer related data, including contractual data detailing Service Level Agreements, type, number, identifiers and characteristics of outsourced equipment. This information is processed by ICFNM in order to define the management scope for each customer and to accordingly configure management applications.

A Trouble Ticketing OSS is the destination to which ICFNM forwards notifications about fault occurrences which result from ICFNM diagnostics activity.

Challenges in interfacing external OSSs are similar to those found at the interface to RNM systems: the variety of data models, APIs, protocols, which require an architecture which would decouple ICFNM from the peculiarities and evolutionary changes of different OSSs. 


\subsection{Organization and processes}

The pressure faced by telecommunication service providers to deal with global competition while rapidly developing new services, is reflected in the level of architectural flexibility required from ICFNM system. ICFNM aims to provide customer with a unique point of contact, the "front office", where help desk operators directly answer queries related to any service trouble.

In addition to the provisioning of a unique point of contact, ICFNM aims to off-load "back office" personnel by offering the front office personnel knowledge about service configuration, utilization parameters and faulty conditions while hiding complexity of networks and equipment. With powerful analysis tools help desk operators are able to direct technical personnel to the problem area in a majority of the cases.

ICFNM deployment configurations need to match PTO organizational processes. For that reason an architecture of ICFNM should foresee a hierarchy of operation centers distributed over the territory. Time-dependent management domains can be assigned to operation centers and individual operators, in terms of supported customers, managed network technologies and functional competencies.

\section{ARCHITECTURE}

This section describes the architecture of the ICFNM system in terms of functional capabilities, physical aspects and platform issues. The relevant choices with respect to the enabling technologies used are also discussed.

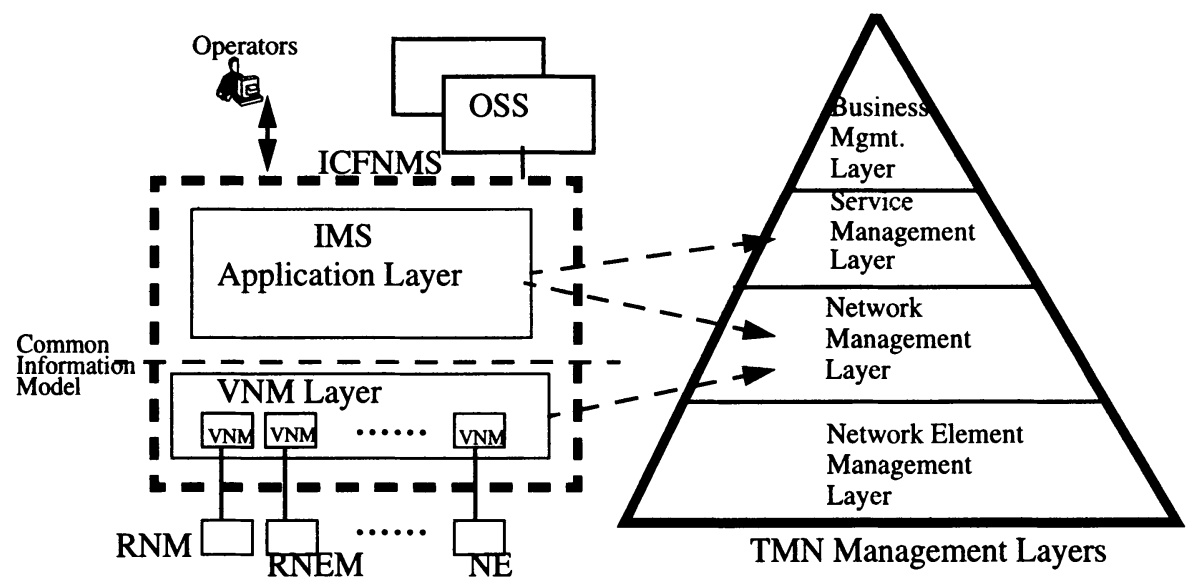

Figure 3 Architecture layering.

\subsection{Functional architecture}

The functional architecture aims to describe and organize the functional capabilities of the ICFNM system by: defining the logical grouping of ICFNM functionality and map- 
ping ICFNM functionality to a widely accepted telecommunication management framework (TMN).

The characterization of the context where ICFNM is positioned highlights the challenges to design a system capable of coping with the evolution of telecommunication services, technologies and organizations. This has lead to the main architectural choice made, i.e., the recognition of a three layered architectural approach (see figure 3):

- the RNM layer, with the purpose of managing the "real" NE and networks

- the VNM layer, with the purpose of applying customer focused filters to the overall set of management information available at the RNM layer for a particular network technology

- the IMS Applications layer, with the purpose of operating the set of virtual, integrated networks that provides telecommunication services to a specific customer.

The layered architectural approach allows for the handling of the system complexity by implementing the normalization of management data and virtualization of networks at the VNM layer while achieving integration of management data and functions at the IMS layer.

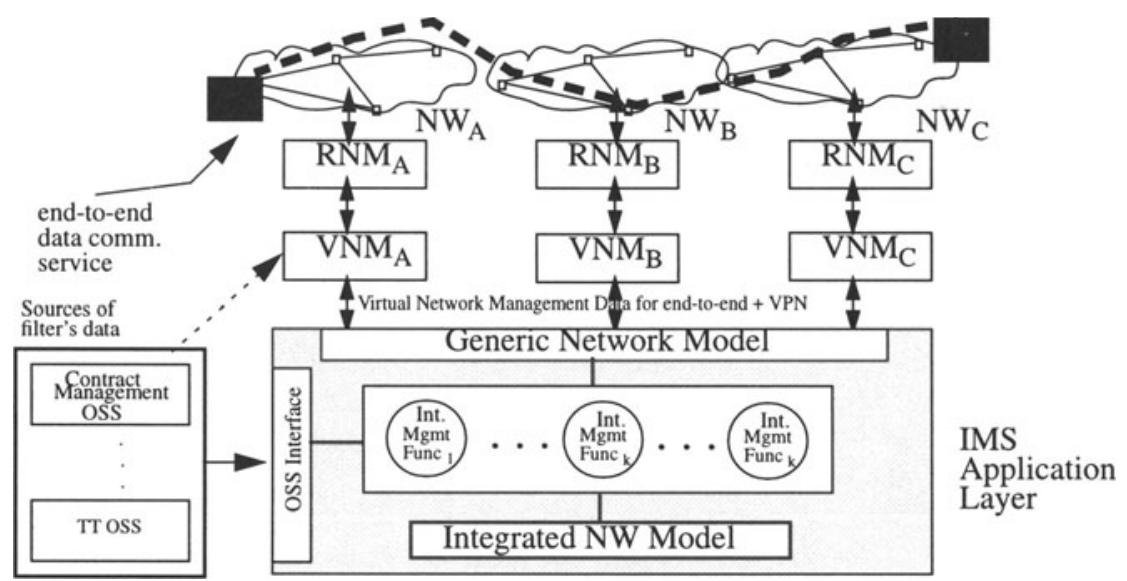

Figure 4 IMS Application layer management functions.

\section{ICFNM: Virtual network management layer}

This architecture layer fulfills two major objectives: normalization and virtualization with respect to network management information.

Normalization: in order to operate the integrated network, ICFNM needs to interface to different entities such as NEs, RNEM and RNM, thereby supporting a wide range of interfaces, generally characterized by different syntaxes, information models, services and behaviors.

Data available at the various RNM interfaces is not suitable for being directly used by the IMS Application layer because of the differences in the expressiveness of management data which are made available through their MIBs. Some MIBs are too fine grained, addressing details not relevant to ICFNM (e.g., network internal not relevant to 
service configurations). Other MIBs do not provide enough information for ICFNM (e.g., because no explicit association exists between network resources and customers using the resource).

In order to control the complexity of the managed systems, we decided to introduce the notion of a Virtual Network Management (VNM) layer. The VNM layer hides behavioral, protocol and modeling peculiarities of the different managed technologies by mean of:

- management protocol conversion to a common management protocol

- information model conversion to a Generic Network Model

- a set of management capabilities (e.g. threshold monitoring, polling, filtering) that apply to all network technologies and hide ICFNM applications from behavioral peculiarities.

Virtualization: the VNM layer offers to the ICFNM application layer (i.e., the IMS) a virtualization of the real network by generating virtual views of the management data available from the RNM.

The functions which generate virtual views use data typically provided by external OSSs (see also section 2.4). For example:

- Customer Ids to be associated with virtual network items provided by Contract Management OSS

- identifiers of network resources assigned to a customer (e.g., routers, interfaces, etc.) provided by Contract Management OSS and Service Provisioning OSS

- identifiers of network resources used by a given service (e.g., permanent virtual circuits) provided by Service Provisioning OSS.

When designing and tuning VNM, a key performance issue is deciding on the technical alternatives for filter application and construction of virtual views. This operation requires access to RNM MIBs, extraction of OSS data and computation of a large amount of information proportionally to the sizes of managed networks. Typical examples of architectural alternatives are:

- compute virtual network views on-demand as required by a management function. This alternative is feasible either when the response time is not a critical factor or when the OSSs and RNMs have close to real time response times. ICFNM applies this approach primarily for extracting up-to-date status information from the RNMs.

- store virtual network views persistently, by computing an initial value for it and keeping it up-to-date by handling asynchronous notification of RNM or OSS data changes. This alternative minimize the load on the RNMs and OSSs but requires them to support asynchronous change notifications. ICFNM adopts this approach for handling network configuration data.

- trigger periodical recomputation of the view and persistently store the result. ICFNM adopts this approach for collecting performance statistics on NE's and network resources.

Identification of the most suitable approach requires a trade-off analysis among the design simplicity allowed by the first option, the response time optimization offered by the second one and the increased computational load on the RNMs caused by the third 
one. Optimal balance between performance, load and complexity is a complex architectural issue. Indeed OSSs and RNMs services impose constraints thereby allowing only for sub-optimal solutions (for example, certain commercial RNMs do not support extensive polling of MIB attributes therefore requiring applications to perform limited polling and integrate this data with asynchronous notifications).

\section{ICFNM: IMS Application layer}

The ICFNM Application Layer uses the services offered by VNM (see figure 4) to achieve the ICFNM main objective, that is to monitor, ensure, and report the Quality of Service offered to customers consistently with contractual data.

Functionalities in the area of Fault, Performance and Configuration Management are supported and summarized in Table 1. Network resources in the context of ICFNM Application layer are those filtered by VNM and relevant to the customer being observed.

Certain key management functions, for example, status monitoring of the end-to-end data communication service spanning across several network domains, cannot be achieved within a single network domain. At the single domain level, in fact, there is no notion of higher level services.

Table 1 ICFNM Functional scope

\begin{tabular}{|l|l|}
\hline Mgmt Svc Area & \multicolumn{1}{c|}{ Management Function } \\
\hline Fault Manage- & $\begin{array}{l}\text { End-to-end monitoring of communication services across multiple technologies } \\
\text { ment } \\
\text { Stanatus monitoring of network resources. } \\
\text { Alarm collection, filtering, and correlation in order to identify faulty components } \\
\text { during "alarms storms" caused by disruption of services spanning multiple net- } \\
\text { works. } \\
\text { Automated diagnostics in order to locate and isolate fault conditions. } \\
\text { Automatic generation and closure of Trouble Tickets. } \\
\text { Proactive fault management. }\end{array}$ \\
\hline $\begin{array}{l}\text { Performance } \\
\text { Management }\end{array}$ & $\begin{array}{l}\text { Monitoring, recording, and reporting on availability at service and network } \\
\text { resource level. } \\
\text { Monitoring, recording, and reporting on end-to-end connection quality. } \\
\text { Identification of QoS level and variations. }\end{array}$ \\
\hline $\begin{array}{l}\text { Configuration } \\
\text { Management }\end{array}$ & $\begin{array}{l}\text { Generation and maintenance of the configuration of the integrated multi-domain } \\
\text { customer network. } \\
\text { Population of the configuration data through network autodiscovery, access to } \\
\text { external OSSs (e.g., Customer Contract OSS) and operator input. } \\
\text { Support for configuration queries and report generation. }\end{array}$ \\
\hline
\end{tabular}

For example, the status monitoring of an end-to-end connection (see figure 4) will require the monitoring of the status of each $\mathrm{NE}$ and each service element belonging to the virtual view for that service, with respect to a particular customer.

Therefore, in order to manage customer specific telecommunication services provided on technologically diverse networks, the IMS Application layer integrates virtual- 
ized network data extracted by VNM from the RNMs.

The operation of mapping a service to its components available at VNMs layer can only be performed if topological information on the "integrated" network is available to the ICFNM system. This is accomplished by persistently maintaining an Integrated Network Model at the IMS Application Layer. The design of the Integrated Network Model is the key architectural issue of the IMS application layer and is a trade-off solution between:

- generality, to increase the system ability to easily integrate new networks

- expressiveness, to increase the system ability to support new management functions.

The chosen approach is based on an Object Oriented model of the integrated network. It achieves a balance between generality and expressiveness of the model by means of an interplay of classification and specialization techniques.

\subsection{ICFNM physical architecture}

The selection of a proper physical architecture needs to cope with different requirements, in terms of organizational processes, scalability and availability of the system.

The system needs to scale efficiently with the size of managed networks, be flexibly deployed into a distributed operational environment and be resilient to system faults. This permits optimized system deployment depending on networks size, characteristics of managed resources and traffic load patterns.

Different physical architectural options have been taken into account when designing ICFNM:

- Centralized ICFNMS: one Workstation(WS) hosts IMS and all VNMs

- Semi-distributed ICFNMS: a centralized Workstation hosts IMS, while VNMs are distributed over multiple WSs interconnected either locally with IMS or remotely with RNEM / RNM systems according to traffic patterns

- Fully-distributed ICFNMS: additionally to VNMs distribution, all different IMS applications are distributed over different interconnected workstations.

A thorough analysis concluded that a fully-distributed ICFNMS was the most suitable solution matching the driving requirements. In a fully-distributed ICFNM, VNMs, and IMS Applications may be freely distributed over multiple workstations interconnected via LANs and/or WANs thus permitting optimized and flexible system deployment.

\subsection{ICFNM: Platform and enabling technologies}

This section reviews the most critical components of the ICFNM development and run time platform in terms of relevant commercial products and enabling technologies chosen for the ICFNM system.

\section{Platform components}

The design of a fully distributed system requires the selection of appropriate enabling technologies based on run-time and development considerations. For reasons described 
below, our choice was for the Common Object Request Broker Architecture (CORBA) (OMG, 1995). We selected IONA's Orbix implementation.

Specifically, CORBA Interface Description Language (IDL) was adopted to describe IMS interface to VNM layer as well as IMS internal object interfaces.

CORBA provides for a key part of our ICFNM communication infrastructure, complemented in the first release of the system by SNMP traps to support asynchronous communication. This was done because CORBA Event Services, while already defined by the OMG, had no product level implementation available at the design time for the first release of the ICFNM system.

Oracle Relational Database - interfaced by applications via an object encapsulation layer - HP OpenView Network Node Manager, X11R5, Motif were adopted as additional key elements for the definition of the ICFNM development and run time platform.

\section{Rational for selecting the CORBA enabling technology}

The choice of CORBA has a significant architectural impact on the entire ICFNM system. The architectural perspectives presented below allow us to share practical experience of an early CORBA-technology adoption in the application domain of Integrated Service and Network Management.

a. Information modelling perspective.

The industry has acknowledged that Object Orientation provides the most suitable paradigm for managing network and system resources as well as for designing distributed applications. Notably OMG CORBA is gaining a growing consensus as a leading architecture for object distribution. CORBA technology can be effectively applied in the context of Network Management since it allows management systems to be aligned with the evolution of the computing world.

A joint OMG/TTU task force is defining a CORBA based architecture for TMN applications and addressing topics such as mapping GDMO and SNMP macros to IDL as well as mapping IDL into GDMO (Rutt, 1996).

Our experience proved CORBA IDL to be a powerful language for defining information models (comparable with GDMO and superior to SMI (Ashford, 1993)), and, in general, for application object interfaces.

The use of IDL allows for a clear separation between interface definition and implementation, thus completely decoupling the development of clients and servers. This allows the freedom, for instance, of using different languages like $\mathrm{C}++$ and Smalltalk, and running on different operating systems. While this was not required in the first release of our system based on HP Workstations and HP-UX, we expect system evolution to require applications to be based on different platforms.

b. Language implementation perspective.

While separation between interface definition and implementation is also a key characteristic of GDMO / ASN.1, CORBA provides a much simpler solution to the same problem and it doesn't require designers to be familiar with complex abstract syntax notations or encoding/decoding schemes. IDL syntax is basically an extension to $\mathrm{C}++$ and has been conceived with the goal of achieving a simple integration 
with implementation languages, allowing the mapping to $\mathrm{C}++$ as well as to other languages.

CORBA also maintains the Object Orientation paradigm of TMN, supports Managed Object Classes and Managed Objects (the instances), and effectively supports inheritance to allow extensibility, evolution and specialization of object properties.

c. Communication perspective.

From a communication perspective, CORBA allows a reduced communication overhead. Multiple naming contexts are endorsed where object locations are transparent to applications. This leaves the ORB charged with the localization of the proper object implementation, the implementation activation, the delivery of the method invocation and the reporting of results. Different activation policies allow a method base selection of persistency vs temporary activation.

Discovery of object services at run-time provided via a DII (Dynamic Invocation Interface) is an alternative to a static invocation of methods. The potential of DII is quite powerful in the ICFNM application context and allows the management of applications to be decoupled from the underlying technology evolution.

SNMP and TMN worlds foresee a unique management-agent paradigm, and recognize that different roles may be taken by the same application in different contexts. Multiple communication paradigms are supported in addition to the client-server paradigm. Indeed publish-subscribe and peer-to-peer schemes are also available as third party commercial products.

d. Synergy between the development platform and the run-time environment through the product life-cycle.

A main requirement for us was the ability to integrate effectively CORBA into our corporate software engineering computing environment. The OMT Object Oriented Analysis and Design methodology was applied to ICFNM system design, to describe application objects, to define the information models at the IMS-VNM border, the OSSs interfaces, the internal application interfaces and the database.

Adoption of Object Orientation was not limited only to analysis and specification stages, but was also applied to design, implementation, and support of ICFNM applications distribution.

A productive application of CORBA to the analysis and code construction phases was possible thanks to available commercial tools supporting the translation of OMT object models to IDL, and IDL compilers providing the mapping of object interfaces into $\mathrm{C}++$ application skeletons.

e. Platform evolution strategy perspective

CORBA technology is not dedicated to the telecommunication industry. It has been conceived and supported by the computing industry, resulting in lower technology costs at both development (libraries, development tools) and deployment levels (runtime licensing costs). The evolution of CORBA service definition and product availability (e.g., security) will provide a flexible platform on which applications targeting business needs can be built without the need to focus on support functions, possibly integrating software components from third party vendors. 


\section{CONSIDERATIONS ON ARCHITECTURAL QUALITIES}

Deregulation, technology innovation and competition are motivating telecom operators to deploy timely and effectively new networking technologies and products.

Extensibility is a key architectural requirement for the ICFNM system, to allow for a simpler integration of new network technologies as well as for allowing the provisioning of new and modified management services. The Object Oriented approach, as well as the definition of a reusable and customizable virtualization layer (i.e., VNM) and CORBA are key architectural choices sustaining our reach for extensibility as seen in the previous sections.

An additional key architectural requirement for the ICFNM system is scalability. The system needs to cope with an increasing size of managed networks, number of customers, and services while offering adequate performance and response time to system operators, who are distributed across geographical areas. This objective is pursued by designing a fully distributed system, scaling efficiently with the size of managed networks which can be flexibly deployed into a distributed operational environment.

Support for distribution and scalability includes:

- clients of applications interfaced to the users execute on a local operator workstation

- flexible definition of a management domain at an operator level, (for instance, partitioning network domains according to managed Virtual Private Networks and functional criteria)

- definition of VNM classes, with VNM instances assigned to configurable domains and running on different workstations

- IMS application subsystems on different workstations. In case of computation intensive applications, such as of fault correlation and diagnostics processes, granularity of distribution may reach the individual process level, with one workstation allocated to one or several correlation processes.

The desired implementation of ICFNM has resulted in a system which may be freely distributed over multiple workstations interconnected via LANs and/or WANs, thus permitting an optimized deployment depending on networks size, characteristics of managed resources, and load patterns.

\section{EARLY RESULTS}

The first release of ICFNM system has been installed in the field for supporting Telecom Italia's growing needs for managing VPN and Outsourcing customers.

Benchmarks made on our deployed ICFNM system confirm the scalability, flexibility and performance of the system and indicate that CORBA is already a viable solution for distributed object-based telecommunication management systems. Detailed performance data is being collected from the field and processed in order to identify needs for VNM tuning as well as a practical feedback for the development of a new system release at the time of writing this paper.

A major result was the capability of designing and developing the system in an aggressive timeframe. CORBA support to object distribution has been the key factor in 
achieving this result. IDL proved to be immediately understandable to $\mathrm{C}++$ software designers, allowing reduction of the ramp-up time for introduction of the new technology.

\section{CONCLUDING COMMENTS}

Telecommunications management business in the form of Outsourcing is a rapidly growing sector of the industry. Delivery of high quality outsourcing services to business customers at competitive costs requires a sophisticated organizational and technical infrastructure. This paper has introduced the concepts, drivers, and the overall role of Integrated Customer Focused Network Management System - an emerging management technology critical to the outsourcing business.

A technical approach for the development of ICFNM systems based on the objectoriented paradigm has been described. Specifically, this approach utilizes the CORBA development and run time enabling technology.

Our experience in advancing ICFNM technology suggests that the telecommunications industry is facing a number of challenges.

The integration of network management in general calls for an explicit recognition by standards bodies and industry associations (e.g., NMF) of the need to establish effective information models targeted for interconnected heterogeneous (from technology perspective) and hybrid (from ownership perspective) networks.

The further advancement of TMN concepts and standards, with respect to Service Management, is seen as an important enabler of reuse of information models in the development of ICFNM systems, as well as of interoperability among management systems and products within the overall TMN framework.

\section{Acknowledgments.}

We wish to thank V. Bigaroni and E. Pignatelli (Telecom Italia), G. Cortese, F. Macuglia, A. Marconi, and M. Peruso (Sodalia) for their valuable contributions.

\section{REFERENCES}

Ashford, C. (1993) Comparison of the OMG and ISO/CCITT Object Models. Joint NMF/OMG TF on Object Modeling.

Bigaroni, V. Calabrese, M. Long, D. Lumello, N. and Saracco, R. (1992) Toward open network supporting customer control services. Globecom '92. Orlando.

Feldkhun, L. Marini, M. Bigaroni, V. and Pignatelli, E. (1996) Integrated Customer Focused Network Management in a Heterogeneous High Speed Networking Environment (practical experience of Telecom Italia). Interworking'96, Nara, Japan.

Object Management Group. (July 1995) The Common Object Request Broker: Architecture and Specification, Revision 2.0.

Rutt, T. Mayne, R. and others. (1996) CORBA-Based Telecommunication Network Management System, Draft. OMG Telecom Special Interest Group.

Sloman, M. (1994) Network and Distributed Systems Management. Addison-Wesley. 